International Journal of Nursing and Midwifery Vol. 5(1), pp. 6-13, January 2013

Available online http://www.academicjournals.org/ijnm

DOI: $10.5897 / \mathrm{JNN} 11.043$

ISSN 2141-2499 @2013 Academic Journals

\title{
Analyzing the brunt trouble of information and communication technologies in the delivery of reproductive health care service in health centers of Shirvan, North Khorasan, Iran
}

\author{
Kazemzadeh Mohammad Reza ${ }^{1}$ and Esmaeilzadeh Mahdi ${ }^{2 *}$ \\ ${ }^{1}$ Welfare Office of North Khorasan, Welfare Organization of Iran, Iran. \\ ${ }^{2}$ Department of Basic Science, Shivan Branch, Islamic Azad University, Shirvan, Iran.
}

Accepted 16 May, 2012

\begin{abstract}
This study analyzed the potential impact of information and communication technologies (ICTs) in the delivery of reproductive health care services. Twelve health establishments located in the districts under the coverage of the micro-Telco were selected. Of the twelve establishments, only ten were found open at the time of the visit. A total of 15 surveys were personally administered by the author. A questionnaire was designed to identify the reproductive health information needs of health center workers and how ICT could help to satisfy those needs. The analysis of questionnaire data was conducted using the MINITAB v14.2 statistical software application. The results showed that although health center workers believe that the use of ICTs would allow them to have better access to health information and therefore, improve the quality of the health care provided, they fail to see how ICTs could satisfy most urgent needs such as lack of qualified personnel and medical equipment. This study was undertaken to assess the current health information needs of health facilities in the area of reproductive health care; however, similar analyses can be conducted to assess more general health care needs.
\end{abstract}

Key words: Information and communication technologies (ICTs), reproductive health care, Shirvan.

\section{INTRODUCTION}

Information and communication technology (ICT) in the health sector is the application of information technology to facilitate the delivery of appropriate health services to the populace (Mechael, 2005). ICT contributes to health education, knowledge sharing, health monitoring, statistics, the delivery of care, and in meeting internationally agreed health targets, with respect to a number of diseases (United Nations Development

\footnotetext{
*Corresponding author. E-mail: mehdi_dna@yahoo.com.
}

Programme (UNDP), 2003). Its impact in health care has been particularly significant in developing countries and in rural settings, where long distances and the quality of the infrastructure hinder the movement of physicians and patients (Geissuhler et al., 2003), reducing the quality of the health services delivered.

However, despite of the potential contributions of ICTs to health services, some constraints exist that prevent their widespread implementation. Some of the more obvious constraints common to developing countries include, the limits of physical access to new technologies, the high cost of providing such access for the less well 
endowed, and the exclusion from access due to the inadequate capabilities of large sections of the population (Chandrasekhar and Ghosh, 2001).

The focus of this paper is to assess the potential impact of ICT services in the delivery of reproductive health care in health care centers and to evaluate the feasibility of acquiring such services. A United States Agency for International Development (USAID)-funded ICT initiative recently developed in Iran to expand connectivity to health care centers areas is taken as a case study. The pilot project consists on creating a local microtelecommunication enterprise (micro-Telco) that will provide, in the initial phase, internet access and voice communication services (within the local network) to a cluster of health care centers districts in North Khorasan Province. While the basic service package fee will include a maximum time of Internet access, voice communication within the network will be unlimited.

\section{METHODOLOGY}

The study was conducted in December, 2010. At that time, only a small group of users had been connected to the local network, and only voice communication services were available. Twelve health establishments located in the districts under the coverage of the micro-Telco were selected. Of the twelve establishments, only ten were found open at the time of the visit. Due to the small number of health workers available at each health center, all of them were approached and asked to voluntarily participate in the study. A total of 15 surveys were personally administered by the author.

A questionnaire was designed to identify the reproductive health information needs of center health workers and how ICT could help to satisfy those needs. The questionnaire included structured (multiple-option) and unstructured (open-ended) questions. Specifically, the questionnaires gathered information on three topics:

1. Information needs on reproductive health: What kind of reproductive health information the health workers need during their everyday activities and how they currently satisfy those needs; 2. Perceptions about the quality of the existent reproductive health services provided;

3. Perceptions of the impact of ICTs: What they think would be the impact of having/using ICTs in the provision of reproductive health care services.

An additional small survey was designed to identify similarities and differences among health establishments in the management of their health care services. Only the head of each establishment or the person in charge at the moment of the visit responded to this additional survey. Grounded theory approach was used to analyze the qualitative data. The data was small enough (15 surveys) to be coded and categorized manually. Not more than 5 categories were used to categorize each question. The analysis of questionnaire data was conducted using the MINITAB v14.2 statistical software application. Non-parametric (Mann-Whitney) and chi-square tests were conducted to investigate differences across groups and associations between variables whenever the sample size allowed it.

\section{RESULTS}

\section{Characteristics of the health facilities}

The health facilities visited belonged to the category of health posts (HPs) which provide basic health care services such as health promotion, disease prevention and patient recovery. They serve communities with 600 to 3,000 inhabitants. Only three of the HPs visited had one full-time physician each, who usually does programmed short visits ( $2 \mathrm{~h}$ per week) to the closest health posts. Regarding the use of ICTs, eight HPs had one cell phone each, mostly used to contact a physician, an obstetrician or the hospital in North Khorasan during health emergencies. However, due to the price of the cell phone calls, most health workers preferred using the public cabins, as indicated by this participant:

'We have the HP' cell phone but we prefer to use the public phone because it is cheaper and we can explain the (medical) problem better."

Text-messaging was also an option, although it also had limitations:

"We prefer using text messaging because it is cheaper than making phone calls; however, it is difficult to give many details that way....".

For non-emergency cases, personal visits were the preferred choice. Reproductive health promotion and education activities are usually coordinated with key community members and groups, such as mother's committees, school teachers and the municipality. The communication among them also occurred face-to-face. About two thirds (65\%) of the HPs' patients are female. The most common reasons for their visits are family planning and prenatal care. For the latter, personal visits play a crucial role in the delivery of health care. These patients are scheduled to come for their prenatal controls, but whenever they fail to show up, the nurse or obstetrician has to visit the patient at home. This, in turn, leaves the health posts unattended and incoming patients waiting and leaving without being attended to.

\section{Characteristics of the respondents}

The average age of the respondents was 37 years old (standard deviation $(\mathrm{SD})=8.12$ ) and the average years of experience in their current profession was 10.6 years $(S D=6.52)$. Table 1 presents the distribution of the sample according to profession, gender and the position occupied at the health facility. About seventy-five percent of the total sample was female. Chi-square tests 
Table 1. Sample distribution.

\begin{tabular}{lccccc}
\hline \multirow{2}{*}{ Profession } & \multicolumn{3}{c}{ Gender } & \multicolumn{3}{c}{ Position at the HP } & \multirow{2}{*}{ Total } \\
\cline { 2 - 6 } & $\mathbf{F}$ & $\mathbf{M}$ & Manager & No manager & \\
\hline Physician & 1 & 2 & 2 & 1 & 3 \\
Obstetrician & 1 & 2 & 0 & 3 & 3 \\
Nurse & 4 & 0 & 4 & 0 & 4 \\
Health Technician & 5 & 0 & 0 & 5 & 5 \\
Total number of respondents & 11 & 4 & 6 & 9 & 15 \\
\hline
\end{tabular}

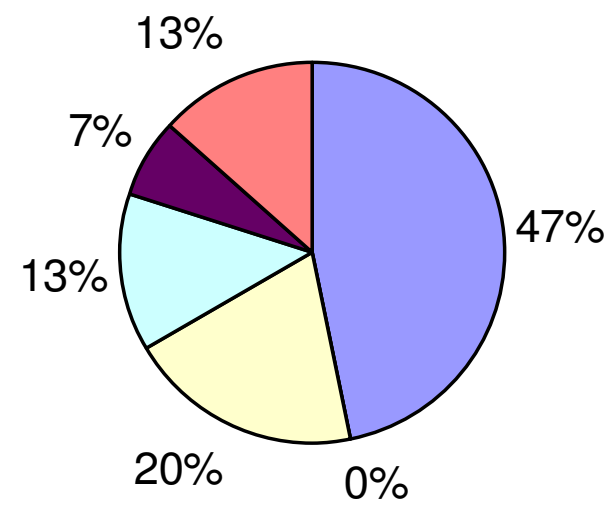

$\square$ Written sources w ithin my health institution

$\square$ Written sources outside my health institution

$\square$ Clinicians w ithin my health institution

Clinicians outside my health institution

Internet

Continuing education courses

Figure 1. Most frequent information sources about reproductive health.

conducted to find associations between characteristics, found associations between profession and position (Chi$\mathrm{Sq}=12.2$, $\mathrm{P}$-value $=0.007)$ and between profession and gender $(\mathrm{Chi}-\mathrm{Sq}=8.2$, P-value $=0.004)$. For the latter, profession were combined into 2 levels: physicians (physicians and obstetricians) and non-physicians (nurse and technicians).

\section{Reproductive health information needs}

The most frequent information needs on reproductive health that the respondents encountered at work are: family planning (40\%), pre-natal care $(27 \%)$ and care of high-risk obstetric patients (20\%). Health workers were asked to indicate their most frequent sources of information about reproductive health. Figure 1 shows the results regarding the information sources used. We can observe that two thirds of the respondents' information sources, including written sources and colleagues are found within their health institutions (written sources and clinicians). No significant differences were found across respondents grouped by gender, profession nor position. Regarding the obstacles to obtain access to reproductive health information, most of the respondents (80\%) indicated 'lack of time' as the first biggest obstacle, and 'not updated information' and 'difficult access to health information' as the second and third biggest obstacles. The same proportion of respondents reported that training courses, if offered, were offered about twice a year at the health network offices, the health regional offices or in the capital country. All clinicians 'would like to receive more courses', especially in topics such as 'care of high risk pregnancy and emergency births' (40\%) and 'pre-natal care' $(20 \%)$. Their own health facility was the location of choice $(80 \%)$ for the training courses, and those who still preferred to travel indicated that it was because of the opportunity to interact with other clinicians when the training was in the Network or Regional offices. Figure 2 shows the main reason for not attending training courses.

The frequency of access to information is displayed in Figure 3. From the responses, we can infer that although the health information health workers receive is usually 


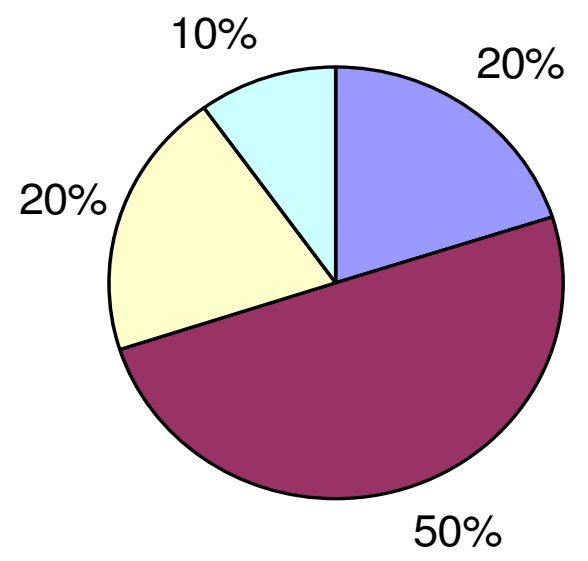

\section{Lack of time}

No one to replace me at work

$\square$ Only heads of health posts or obstetricians attend training Cost

Figure 2. Main reason for not attending training or actualization courses.

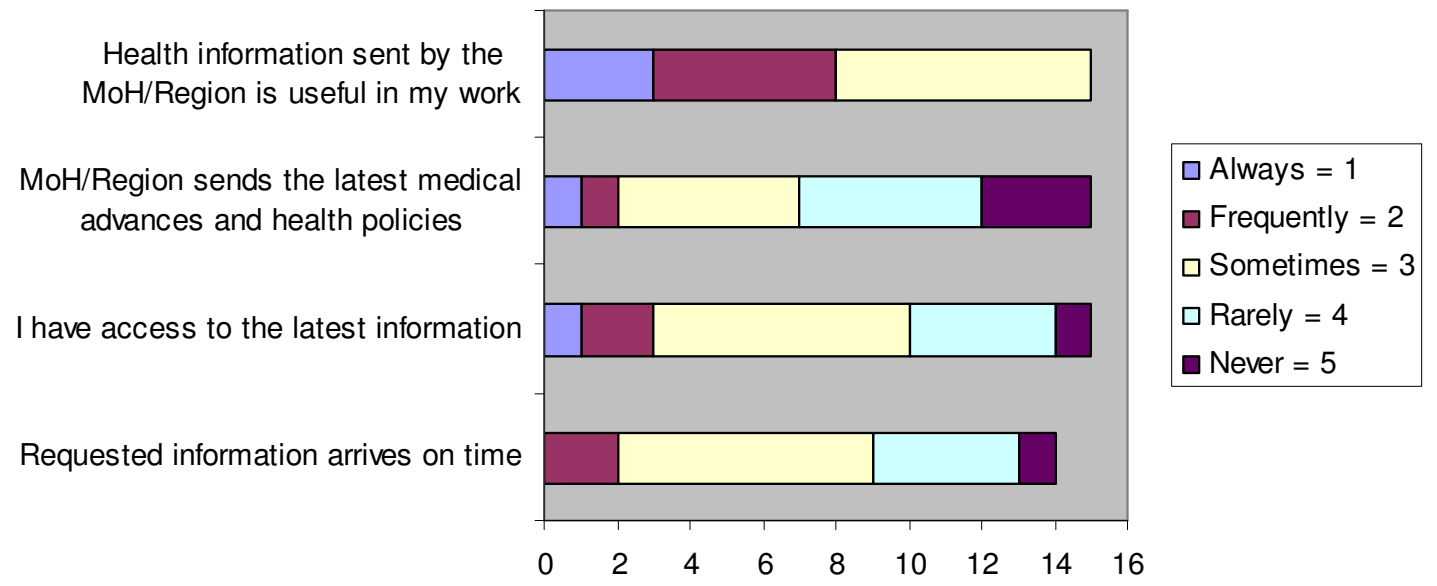

Figure 3. Frequency of access to information about reproductive health.

useful, it does not always arrive on time nor contain the latest information.

\section{Perception of the quality of the existent health services}

In the following section of the survey, participants were asked to give their perceptions about the existent reproductive health services provided by their health establishments. About eighty percent of the respondents considered that the current services satisfied the needs of the community. The quality of the services provided was indicated as 'good' by $53 \%$ and 'acceptable' by $40 \%$.
'Family planning' and 'sexually transmitted diseases (STDs) and uterine cancer prevention' were indicated as the most and the least utilized reproductive health services, respectively. Figure 4 shows the health workers perceptions about what is needed to improve the existent health care services.

\section{Perceived impact of ICTs on the reproductive health services}

The next section of the survey focused on gathering information about the health workers' perceptions regarding the impact of ICTs in the reproductive health 


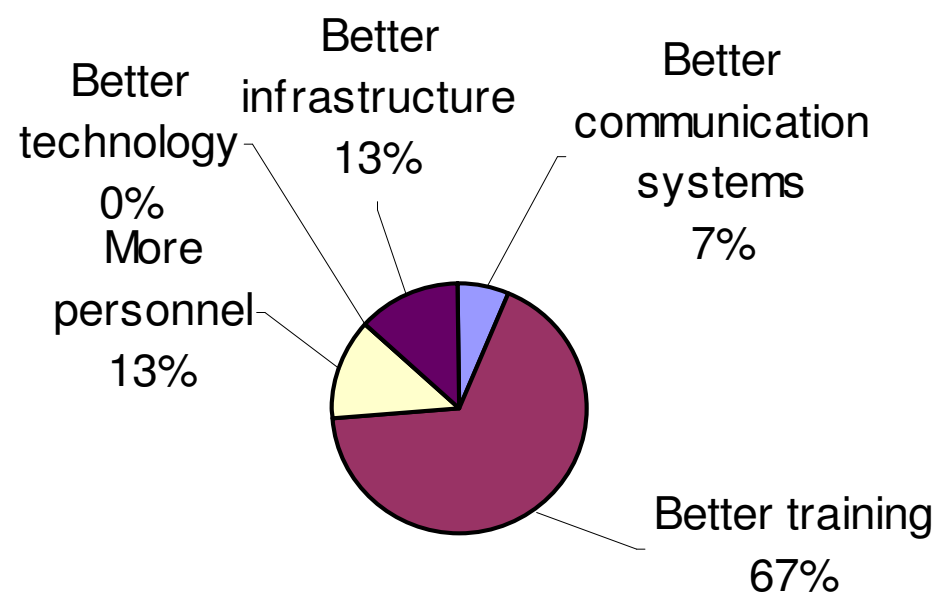

Figure 4. Perceptions of what is needed to improve the quality of the reproductive health services provided (1st priority).

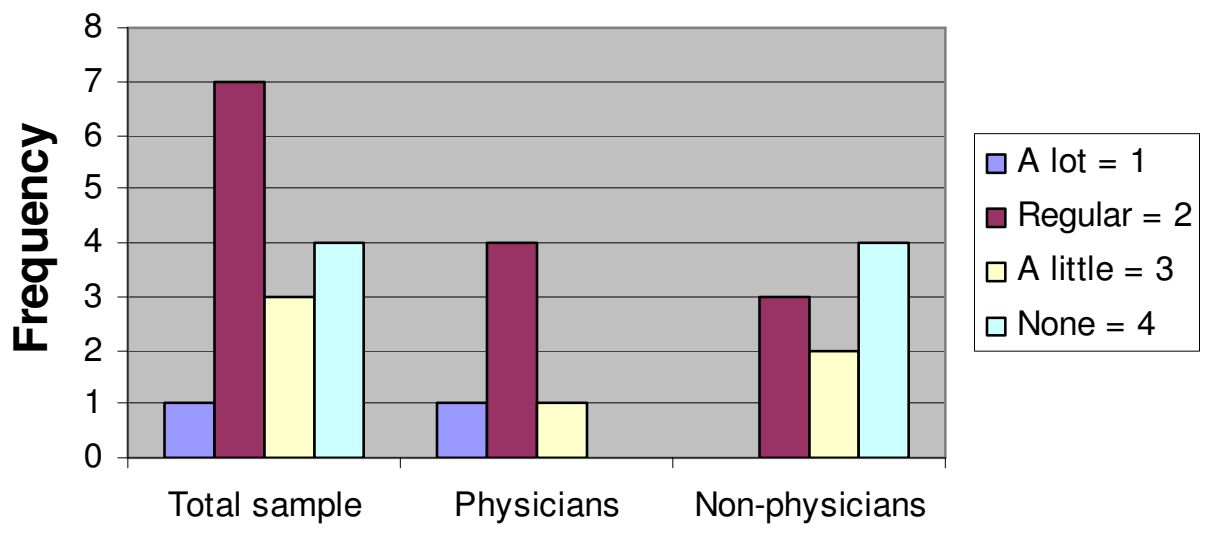

Figure 5. Experience with computers.

services provided. Results show that most of the participants $(80 \%)$ are familiar with some type of ICT, with the cell phone (92\%) being the most used device. Figure 5 shows how experienced health workers are with computers.

A non-parametric test (Kruskal-Wallis) to identify significant differences among groups was conducted. Significant differences were found between the "Physicians" and the "Non-Physicians" group (DF = 1, Pvalue $=0.032$ corrected for ties). The first group had a tendency to be more experienced with computers $($ Median $(M d n)=2)$ than the second one $(M d n=3)$. Of those who had at least "a little" experience with computers, about half of them (55\%) were familiar with Micro soft (MS) Office-type applications. Disregarding their experience with computers, all participants answered that they "would like to learn more about computers and the internet". This enthusiasm was explained by the seventy-three percent of the respondents who indicated that the internet would be the most useful ICT to improve the reproductive health care services. Nonetheless, a Kruskal-Wallis (K-W) test found significant differences between groups with different years of work experience. All of the participants with ' 10 or less years of experience' considered internet as more useful, while those with 'more than 10 years of work experience' considered internet and fixed-line phone equally useful (Figure 6).

Figure 7 shows results about how comfortable health workers would feel using the telephone and the internet in different tasks. No significant differences were found across sample groups. Participants' perceptions about the 


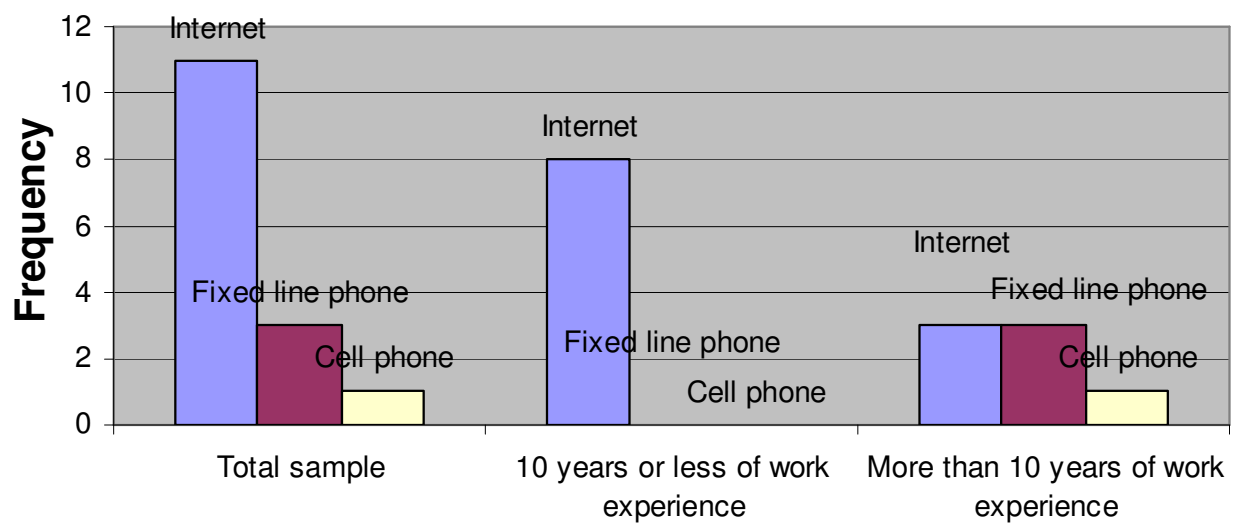

Figure 6. Type of ICT more useful to improve the reproductive health care services.

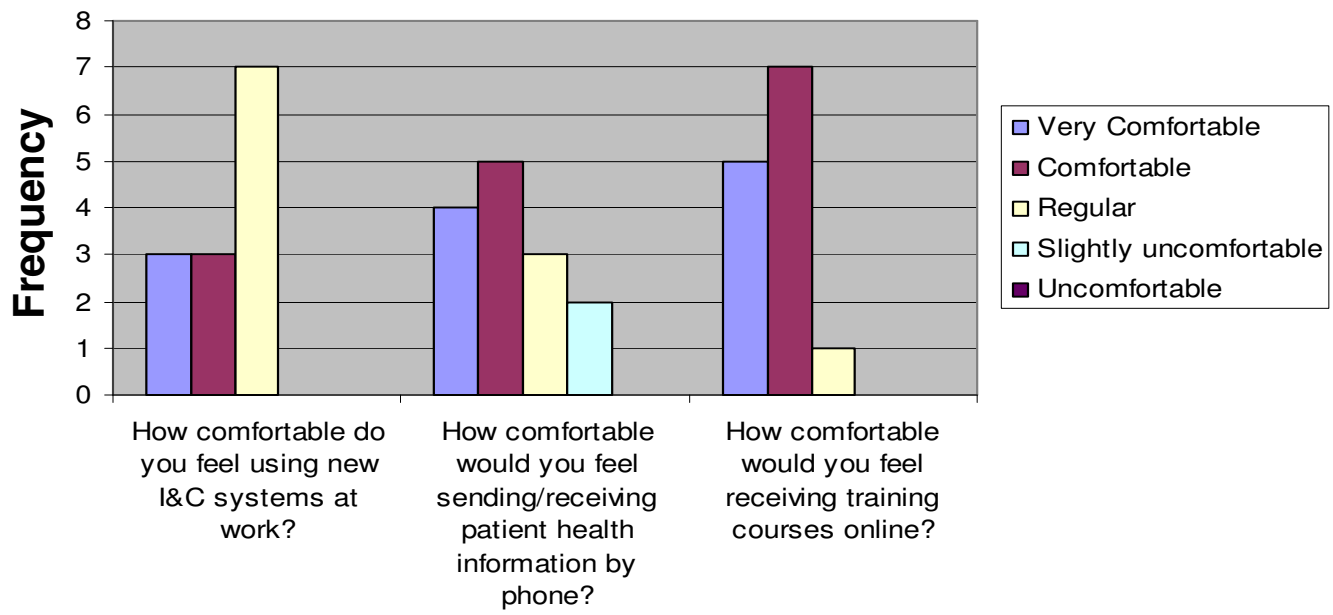

Figure 7. Respondents perception about using ICTs in health care.

impact of ICTs in improving their work were also very positive. Almost all (93\%) responded "Yes" when asked if they considered that using ICTs at work would help them take better medical decisions and improve the quality of health care. The same proportion responded positively when asked if they considered that having better access to medical information due to ICTs would help to reduce medical errors related to reproductive health. Eighty-six $(86 \%)$ of respondent also thought that having real-time access to reproductive health information would make their works easier.

\section{DISCUSSION}

Results indicated that the big majority of health workers in the health facilities were female, especially the nurses and the health technicians. In consequence, genderspecific constraints on medical education and continuing professional development impact disproportionately on these specialties (Geyoushi et al., 2003), and affect the quality of the health care services provided. On the contrary, lack of professional development of health workers and its impact on women's development also becomes a valid concern. In terms of the feasibility of acquiring the services of the micro-telco, the presence of alternative inexpensive communication means, such as public phones and personal visits, appear as obstacles for the acquisition of the aforementioned services. However, unrecognized benefits of the new ICTs, such as increase in patient retention due to better quality of health care services, were not incorporating into the calcu- 
lations. For instance, health workers are aware of the general benefits of using the telephone to coordinate activities with members of the community, but fail to see how this can be profitable or cost-effective for the HPs. Decision-makers should not forget that the effect of ICTs on health differs from its impact on other sectors. Achieving some of the benefits of ICTs requires that health workers are reached and not necessarily the final beneficiaries (Chandrasekhar and Ghosh, 2001), which reduces the investment.

Attending training courses or searching for health information outside the workplace facility implies abandoning the health facility. In this case, online (or offline) distance education would help satisfy this important need. Providing access to reliable health information for health workers in developing countries is potentially the single most cost effective and achievable strategy for sustainable improvement in health care (Pakenham-Walsh et al., 1997) and this can be achieved by the internet. The micro-telco, as a local provider, can help to design and tailor ICT applications to satisfy specific local health information needs. Feedback from local health workers would be crucial in designing application with 'relevant' content. If, for example, an information source indicates a diagnostics test that is not available or affordable in a given setting, then the information given was not relevant (Godlee et al., 2004). Designers have to be careful with this issue, because the frustration that can be caused by continuous access to information that is not relevant can drastically reduce the demand for such services. The lack of communication and cooperation among health care providers outside the community can be improve by the 'unlimited local calls' feature and thus, encourage the sharing of cognitive and even physical resources; although all the HPs belong to the same category of health facility, differences in quality and quantity of equipment, personnel and health care services were found even in HPs not more than 2 miles apart.

Although ICTs can not directly address urgent needs such as lack of qualified personnel or equipment, it can however help to increase the time that the personnel spend in the health establishment and improve the qualifications of the current workforce. Furthermore, policy and decision makers can get a better idea of the current situation of the HPs by having direct interaction with the health workers. Thus, the information generation process and policy making could become an interactive process (Edejer, 2000).

In general, the perceived impact of ICTs is positive. There is a consensus regarding the usefulness of using internet or e-mail in improving the quality of the health care services provided; although, at this point, the specifics of how it would be done are not fully understood. A few health initiatives to improve health care through the use of ICTs were developed with successful results (Martinez et al., 2005). As we gathered from the results of this survey, willingness to learn and use ICTs is not a problem, on the contrary, it is probably one of factors leading to the projects successes.

\section{CONCLUSION}

This study was undertaken to assess the current health information needs of health facilities in the area of reproductive health care; however, similar analyses can be conducted to assess more general health care needs. The positive impact of ICTs in the reproductive health care delivery is clear, but there is still the need to find out how to accurately address the specific needs identified by this research. Regarding financial feasibility of ICTs, the potential increase in the productivity of the health posts could help to cover the expenses of acquiring such ICT services. Further in-depth studies such as detailed costbenefit analyses are required to confirm results from the present study. Even if the health facilities were not able to afford the micro-telco services by themselves, parallel health initiatives involving the use of ICT tools make seem imminent the acquisition of ICTs by the studied health facilities. Therefore, it will be important to keep in mind that current ways of conducting health care activities will have to change in order to better take advantage of the benefits of ICTs. Health workers need to be aware of this fact and be correctly and timely trained, so their current positive perception about ICTs does not change to seeing ICTs as interfering and disrupting of their workflow.

\section{REFERENCES}

Chandrasekhar CP, Ghosh J (2001). Information and communication technologies and health in low income countries: the potential and the constraints. Bull. World Health Org. 79(9)850-855.

Edejer TT (2000). Disseminating health information in developing countries: The role of the internet. Br. Med. J. 321:797-800.

Geissuhler A, Ly O, Lovis C, L'Haire J (2003). Telemedicine in Western Africa: Lessons learned from a pilot project in Mali, perspectives and recommendations. AMIA Annu. Symp. Proc. 2003:249-253.

Geyoushi B, Matthews Z, Stones RW (2003). Pathways to evidencebased reproductive healthcare in developing countries. Br. J. Obstet. Gynecol. 110:500-507.

Godlee F, Pakenham-Walsh N, Ncayiyana D, Cohen B, Packer A (2004). Can we achieve health information for all by 2015 ? Lancet 364(9430):295-300.

Martinez A, Villarroel V, Seoane J, Pozo FD (2005). Analysis of Information and Communication Needs in Rural Primary Health Care in Developing Countries. IEEE Trans. Inf. Technol. Biomed. 9(1):6672.

Mechael PN (2005). Case Study from Egypt: Mobile phones for mother and child care. Available at: http://www.comminit.com/africa/content/mobile-phones-mother-andchild-care.

Pakenham-Walsh N, Smith R, Priestley C (1997). Meeting the information needs of health workers in developing countries. Br. Med. J. 314(7074):90. 
UNDP (2003). Achieving MDGs through ICT: Experiences and Challenges in Viet Nam. Ha Noi. United Nations Development Program. 